\title{
Relation between pressure determined by ophthalmodynamometry and aortic pressure in the dog
}

\author{
William H Morgan, Dao-Yi Yu, Valerie A Alder, Stephen J Cringle, Ian J Constable
}

\begin{abstract}
Aims-Ophthalmodynamometry has been used extensively since the last century; however, controversy surrounds what it actually measures. This study was set up to determine the relation between ophthalmodynamometric (ODP) and systemic blood pressures.

Methods-Aortic pressure was continuously monitored and altered by phlebotomy in six anaesthetised dogs, while ophthalmodynamometry was performed, by directly altering intraocular pressure. Maxillary artery pressure was monitored in two animals. All pressure transducers were zeroed at eye level.

Results-Mean ODP was $96.6 \%(1.6 \%)$ ( $95 \%$ confidence interval, $n=49)$ of aortic pressure. Mean maxillary artery pressure was $95.7 \%(5.5 \%)(95 \% \mathrm{CI}, \mathrm{n}=16)$ of aortic pressure. ODP was $1.9(0.6) \mathrm{mm} \mathrm{Hg}$ $(95 \% \mathrm{CI}, \mathrm{n}=33)$ higher than maxillary artery pressures.

Conclusion-ODP was only slightly below aortic pressure and not significantly different from maxillary artery pressure, the analogue of the internal carotid artery in humans. These results also suggest a retinal artery collapse pressure of at least $\mathbf{1 . 9}$ mm Hg.

(Br f Ophthalmol 1998;82:821-825)
\end{abstract}

Ophthalmodynamometry produces blood pressure information; however, controversy surrounds how this relates to the arterial pressure supplying the eye and brain. It has been claimed to estimate central retinal artery, ${ }^{1-4}$ ophthalmic artery, ${ }^{5-8}$ or internal carotid artery ${ }^{9-12}$ pressure. In spite of its extensive use clinically and for research into ocular 357813 and neurological ${ }^{6-12} 14$ disorders, adequate validation has not been performed.

Clinically, ophthalmodynamometry has been most extensively used for the detection of haemodynamically significant carotid artery stenosis, and has a sensitivity of $71-80 \%$ and specificity of $75-90 \% .^{6912}$ In the past 10 years, duplex carotid Doppler scanning has become much more widely employed principally because of its higher sensitivity (up to $96 \%{ }^{12}$ ). However, ophthalmodynamometry still has some use because of its low cost.

Ophthalmodynamometry is a technique in which the intraocular pressure (IOP) is elevated while observing the central retinal artery (CRA). Diastolic ophthalmodynamometric pressure (ODP) is said to be the minimum IOP at which the first CRA collapse occurs, this collapse being intermittent-that is, pulsating. Systolic ODP is said to be the minimum IOP at which continuous CRA collapse occurs. Different methods are used to raise and measure the IOP, from direct cannulation $^{27}$ to external calibrated compression. ${ }^{1} 810-1315$

A number of authors have speculated upon the relation of ODP to the value of CRA pressure before raising IOP and performing ophthalmodynamometry. Grunwald and Furubayashi $^{8}$ have said that ODP is only slightly higher than CRA pressure. Hedges et $a l^{2}$ concluded that ODP was equal to CRA pressure. Van der Werff, ${ }^{5}$ using a theoretical model of flow from the ophthalmic artery to the CRA, calculated that systolic ODP is 14-17 mm Hg higher than CRA systolic pressure. Others have claimed that ODP is initially equal to CRA pressure for a few seconds before rising to be equal to ophthalmic artery pressure. $^{3}$

Few workers have attempted to verify what ophthalmodynamometry actually measures. In 1926, Duke-Elder, ${ }^{7}$ limited by the technology of the time, by direct cannulation found mean ODP to be $92 \%$ of mean aortic pressure. Only systolic aortic pressure was measured, the diastolic pressure being assumed. Mean pressures were calculated by adding one half the pulse pressure to the diastolic pressure. DukeElder claimed that ophthalmodynamometry estimated ophthalmic artery pressure. $\mathrm{He}$ justified this claim by citing the hydrodynamic principle, that pressure in an occluded vessel is equal to that of the nearest upstream branch point. Under conditions of ophthalmodynamometry, the CRA and choroidal circulation are occluded within the eye, so that the exact branch point within which the pressure theoretically corresponds to ODP is not clear. Hedges $e t \mathrm{al}^{2}$ measured ophthalmic and femoral artery pressure while performing ophthalmodynamometry in the rhesus monkey. They found that ODP and ophthalmic artery pressures were in the same range, but were 16-25 $\mathrm{mm} \mathrm{Hg}$ lower than femoral artery pressure. Others claim that within the carotid artery there is already a drop from aortic pressure of $20-25 \% .^{16}$

In other vascular beds, relatively little flow resistance down the arterial tree is encountered, with little accompanying pressure drop until arterioles with $100 \mu \mathrm{m}$ wide lumens are reached. ${ }^{17}$ In cat mesentery the pressures in $120 \mu \mathrm{m}$ wide arterioles is $80 \%$ of systemic 


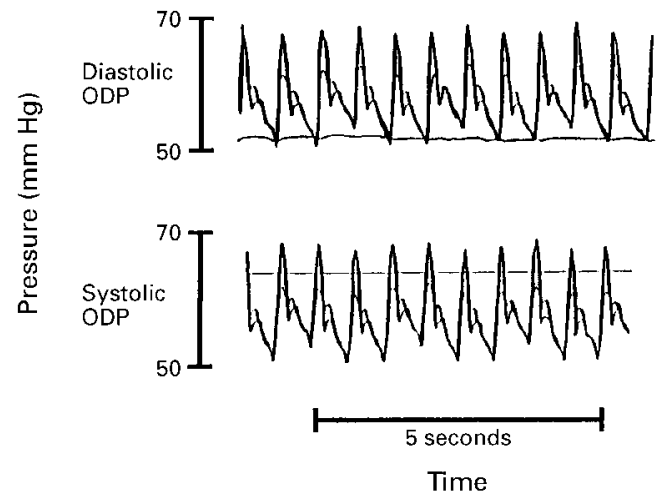

Figure 1 Typical traces of aortic and maxillary artery (max A) pressures. First $C R A$ pulsation (diastolic ODP) was seen when IOP was elevated to a level equal to aortic and maxillary diastolic pressure. Complete CRA closure (systolic ODP) was seen when IOP was elevated to a level above maxillary artery but below aortic systolic pressure.

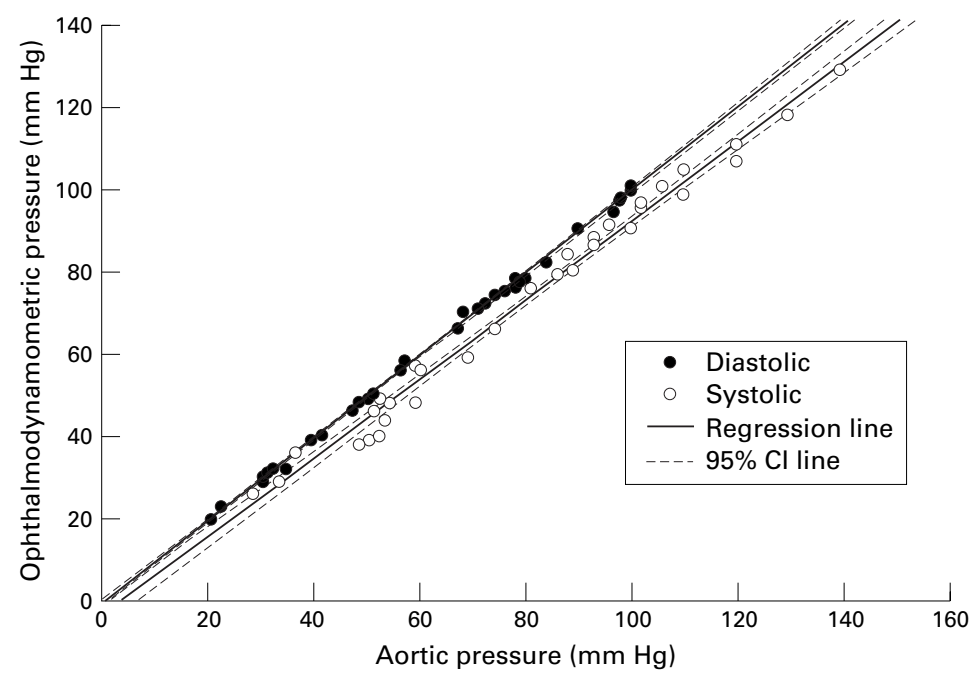

Figure 2 Ophthalmodynamometric (ODP) diastolic versus aortic diastolic pressure and ODP systolic versus aortic systolic pressure, with regression and $95 \%$ confidence interval lines. It can be seen that diastolic ODP is almost equivalent to aortic diastolic pressure, and that the systolic ODP is slightly less than aortic systolic pressure.

pressure. ${ }^{18}$ Central retinal artery diameter has been measured at $140 \mu \mathrm{m}$ and the ophthalmic artery diameter measured at $750 \mu \mathrm{m} .^{5}$

It was this background of confusion concerning what ophthalmodynamometry actually measures, which led us to design the following experiments in order to determine how ODP is related to aortic pressure. We used an in vivo animal model so that the eye and aorta could be directly cannulated, allowing both IOP and aortic pressure to be directly measured and varied over a large range, eliminating the calibration problems that external ocular compressive devices induce. From these measurements we hoped to infer the relation between ODP and internal carotid artery and CRA pressure.

\section{Methods}

ANIMAL PREPARATION

Six mixed breed dogs were used in accordance with the Australian National Health and Medical Research Council animal ethics committee code of practice. The animal set up was the same as has been described previously, ${ }^{19}$ with the following differences. The animals were anaesthetised using halothane inhalation for induction only, followed by a continuous intravenous infusion of a 50:50 mixture of pentobarbitone and thiopentone through the femoral vein, while being ventilated with nitrogen $80 \%$ and oxygen $20 \%$.

The animals were suspended in the prone position with the head held in a maxilla bite clamp. The aorta was cannulated via the femoral artery. The left anterior chamber was cannulated and connected to a pressure transducer. A second cannula was inserted into the anterior chamber, being connected to Krebs solution reservoirs allowing IOP to be controlled.

In two animals the maxillary artery was cannulated as it came out of the infraorbital foramen to form the infraorbital artery. The infraorbital artery is the end artery of the maxillary artery. The cannula tip was inserted pointing directly against maxillary artery blood flow. This cannula was connected to another pressure transducer.

All pressure transducers (Cobe, Arvada, CO, USA) were connected via conditioning modules (Analog Devices, USA) to a Yokogawa (Tokyo, Japan) LR8100 chart recorder and thus femoral artery, vein, and intraocular pressures were continuously recorded. All transducers were held and calibrated at the level of the left eye, so that all pressures are measured with respect to this location. Note that the maxillary artery, being similar to the internal carotid artery of humans, gives rise to the ophthalmic artery.

A planoconvex contact lens was applied to the left cornea. The left retina including retinal arteries were observed with an operating microscope (Zeiss OPMI-1, Oberkochen, Germany).

\section{OPHTHALMODYNAMOMETRY}

Ophthalmodynamometric pressures were determined as follows. The IOP was elevated rapidly to $80 \%$ of aortic diastolic pressure over 3-5 seconds by pressurisation of the Krebs solution connected to the anterior chamber. The IOP was then elevated more slowly, over approximately 20 seconds while the retinal arteries on the optic disc were observed, until diastolic ODP was recorded. The lowest IOP at which complete collapse of the retinal arteries on the optic disc was observed was recorded as diastolic ODP. The retinal arteries were seen to pulsate at this level because the collapse was intermittent. IOP was elevated slowly until the collapse became continuous throughout the cardiac cycle. The lowest IOP at which

Table 1 Means of the ophthalmodynamometric versus aortic pressure linear regression results in six animals

\begin{tabular}{|c|c|c|c|c|c|c|}
\hline & Slope & $\begin{array}{l}\text { Slope } \\
\text { CI }\end{array}$ & $\begin{array}{l}y \\
\text { Intercept }\end{array}$ & $\begin{array}{l}y \\
\text { Intercept } \\
\text { CI }\end{array}$ & $r$ & $n$ \\
\hline Diastolic & 0.971 & 0.030 & 1.11 & 1.96 & 0.999 & 50 \\
\hline Systolic & 0.956 & 0.077 & -3.99 & 7.32 & 0.993 & 49 \\
\hline Mean & 0.967 & 0.043 & -0.68 & 3.28 & 0.998 & 49 \\
\hline
\end{tabular}

The $95 \%$ confidence intervals (CI) were calculated from the six linear regression results. The correlation coefficients were calculated from all the data grouped together. The total number of data points is given in $\mathrm{n}$ column. 
Table 2 Means of the maxillary artery versus aortic pressure linear regression results from two animals

\begin{tabular}{lccll}
\hline & Slope & y Intercept & $r$ & $n$ \\
\hline Diastolic & 0.929 & 1.71 & 0.998 & 16 \\
Systolic & 0.895 & -1.43 & 0.985 & 16 \\
Mean & 0.899 & 1.63 & 0.994 & 16 \\
\hline
\end{tabular}

The correlation coefficients (" $r$ ") were calculated from " $n$ " number of grouped data points.

continuous collapse occurred was recorded as systolic ODP. From the chart recording, while the ophthalmodynamometric criteria were met, the means of the diastolic and systolic pressures over 10 seconds were calculated and recorded. Similarly, the maxillary artery pressures were recorded.

Following systolic ODP measurement, the IOP was brought back to $20 \mathrm{~mm} \mathrm{Hg}$. Phlebotomy was performed in order to lower the blood pressure. The aortic pressure was allowed to stabilise over 3-4 minutes, during which time some hyperaemia of the optic disc was noted. This hyperaemia partially settled before repeat ophthalmodynamometric pressure measurements were performed. The range of aortic pressures were from 20 to $140 \mathrm{~mm}$ Hg. Successive phlebotomies and ODP measurements were performed until the aortic pressure reached 20-30 $\mathrm{mm} \mathrm{Hg}$. The animal was then killed with a lethal injection of pentobarbitone.

\section{Results}

A typical trace of aortic and maxillary artery pressures is shown in Figure 1. First CRA pulsation (diastolic ODP) was seen when IOP was elevated to a level equal to aortic and maxillary diastolic pressure. Complete CRA closure (systolic ODP) was seen when IOP was elevated to a level above maxillary artery but below aortic systolic pressure. The diastolic and systolic ODP did not vary in any measurements, and in particular, no ODP was seen to rise during any measurement.

Figure 2 shows the recordings of diastolic ODP versus aortic diastolic pressures and

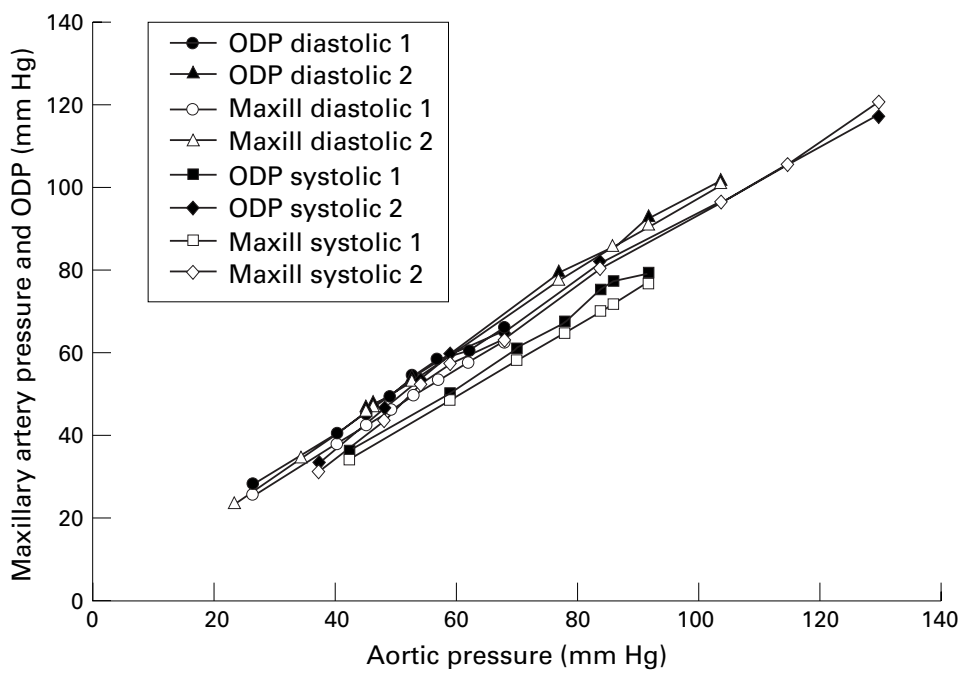

Figure 3 Ophthalmodynamometric (ODP) and maxillary artery (maxill) pressures versus aortic pressure in two animals. The aortic pressure was varied by phlebotomy. Systolic and diastolic pressures are shown. Both systolic and diastolic maxillary artery pressures are seen to be slightly less than $O D P$. systolic ODP versus aortic systolic pressures. It can be seen that diastolic ODP is almost equivalent to aortic diastolic pressure, and that the systolic ODP is slightly less than aortic systolic pressure.

Mean pressures for each recording were calculated as diastolic + (systolic - diastolic) $\div 3$. The linear regression results of ODP versus aortic pressures are given in Table 1. Student's two tailed $t$ test was used to calculate $95 \%$ confidence intervals for the slope and y intercept.

One can see that all ODPs have a very strong linear relation with their aortic pressure counterparts. ODP is almost equivalent (97\%) to aortic diastolic pressure. The systolic ODP is $96 \%$ of systolic aortic pressure and mean ODP is $97 \%$ of mean aortic pressure.

The relation between maxillary artery and aortic pressures (Table 2) was similar to that between ophthalmodynamometry and aortic pressures. Both systolic and diastolic ODP were slightly greater than the respective maxillary artery systolic and diastolic pressures (Fig 3). The difference between ODP and maxillary artery pressure was calculated for each measurement, the $95 \%$ confidence interval calculated for the average of the difference in each animal using Student's two tailed $t$ test. The mean difference between diastolic pressures was 2.0 (0.9) $\mathrm{mm} \mathrm{Hg}(95 \% \mathrm{CI}, \mathrm{n}=17)$, and between systolic pressures was 1.9 (1.1) $\mathrm{mm} \mathrm{Hg}(95 \% \mathrm{CI}, \mathrm{n}=16)$. Combining the diastolic and systolic pressure differences resulted in a mean difference of $1.9(0.6) \mathrm{mm}$ $\mathrm{Hg}(95 \% \mathrm{CI}, \mathrm{n}=33)$.

\section{Discussion}

Mean ODP was found to be $97 \%$ of mean aortic pressure. There is known to be a $2.5 \%$ drop in mean pressure along the whole length of the aorta in dogs, ${ }^{20}$ with relatively little drop in mean blood pressure until $100 \mu \mathrm{m}$ arterioles are reached. ${ }^{17}$ The tapering of the elastic arterial tree, combined with its relatively low flow resistance compared with the microcirculation, is thought to allow for the small loss of pressure, and retention of a large pulse pressure wave throughout the arterial tree. ${ }^{20}$

Our results are in agreement with these circulatory principles, and are similar to those of Duke-Elder ${ }^{7}$ who was limited technically to measuring aortic systolic pressure only. Using his mean pressure definition, our mean ODP was $96 \%$ of aortic pressure compared with his $92 \%$. It is unlikely that the induced optic disc hyperaemia significantly altered the aortic or maxillary artery pressures, as the proportion of blood flow through these vessels to the eye is very small. These results are consistent with experiments in humans ${ }^{1}$ finding no significant difference between ODP and brachial artery pressure when the upper arm and cuff are held at eye level. Our results are at variance to those of Robinson et $a l{ }^{13}$ who related ODP to brachial artery pressure while varying the systemic blood pressures in three sitting humans, finding a slope of 0.61 with a y intercept of $-8.8 \mathrm{~mm} \mathrm{Hg}$. If ODP is similar to 
systemic arterial pressure, one would expect to see a slope close to 1.0 , and a y intercept similar to the hydrostatic pressure drop between the eye and brachial artery cuff, as a result of gravity. Their technique involved the use of a Balliart external compressive ophthalmodynamometer, which has been shown to underestimate the induced IOP, ${ }^{15}$ and hence may partly explain the apparent dissimilarity to our results. This also illustrates the problem of calibration in external compression ophthalmodynamometers. Our results highlight the importance of noting the vertical height difference between brachial artery cuff and eye when performing ophthalmodynamometry clinically. The hydrostatic pressure effect of this height difference should be subtracted from the brachial artery pressure reading to allow a meaningful comparison between ODP and blood pressure to be made. Ocular pneumoplethysmography, a related technique in which a suction cup is applied to the eye, to alter and measure IOP, while an external pressure transducer detects initial ocular vascular pulsation, has been shown to produce measurements with a mean difference of $4.6 \%$ from carotid artery pressure in humans. ${ }^{21}$

In the dog the ophthalmic artery branches off the maxillary. Both the diastolic and systolic ODP were found to be slightly higher than maxillary artery pressure. In order to occlude the retinal arteries, IOP must be raised above the CRA intraluminal pressure and also overcome the transmural pressure required to collapse the vessel. The ophthalmic artery branches off the maxillary artery and its major branches, the anterior and posterior ciliary arteries mainly supply the retinal and choroidal circulation as well as the rectus muscles. Blood in the ophthalmic artery still flowing to non-ocular orbital structures such as the rectus muscles during ophthalmodynamometry is likely to have a pressure less than that of the maxillary artery. Hence, the transmural pressure required to collapse the artery will probably be greater than the $1.9 \mathrm{~mm} \mathrm{Hg}$ mean difference between ODP and maxillary pressures measured here. The transmural pressure required to collapse the brachial artery has been estimated theoretically to be $2 \mathrm{~mm} \mathrm{Hg} .^{22}$ The retinal artery collapse pressure has not been previously measured, although Best et $a l^{23}$ estimated the critical closing pressure of the whole ocular vascular bed to be $6 \mathrm{~mm} \mathrm{Hg}$. The analogue of the maxillary artery in humans is the internal carotid artery, so it is likely that ophthalmodynamometry gives pressure results close to or just above those within the internal carotid artery.

It is reasonable to assume that the retinal and optic disc vasculature will dilate in response to the induced ischaemia during ophthalmodynamometry. A dilatation downstream from the large retinal arterioles will tend to lower the retinal artery pressure. Direct measurements of retinal artery pressure in dogs with micropipettes, ${ }^{24}$ over a range of aortic blood pressures but with a steady mean IOP of 19 $\mathrm{mm} \mathrm{Hg}$, have shown that it is $72 \%$ of systemic blood pressure. Thus, in the dog, a far greater pressure drop from the aorta to the retinal arteries is found, suggesting that at least $25 \%$ of the total pressure drop occurs from the maxillary artery to the retinal artery on the retinal surface where it is approximately 100 $\mu \mathrm{m}$ in diameter. Together, these results show that ODP is quite different from retinal artery pressure.

If we extrapolate to the human situation, then in normal healthy subjects, ophthalmodynamometry can be expected to give results similar to internal carotid artery pressure. These pressures are only 3\% below aortic pressure and hence ophthalmodynamometry should give results similar to systemic blood pressure, allowing for the height difference between the eye and brachial artery. A major problem limiting the clinical utility of ophthalmodynamometry is the accurate measurement of IOP while it is elevated. In spite of this problem ophthalmodynamometry and related techniques remain useful for detecting carotid artery stenosis and significant cross filling via the circle of Willis in cases of carotid artery stenosis. ${ }^{10}{ }^{1425}$ In normal subjects, ophthalmodynamometry is no more useful in estimating retinal artery pressure than measuring systemic blood pressure.

This study was supported by the Ophthalmic Research Institute of Australia, Raine Medical Foundation, and the National Health and Medical Research Council of Australia.

The authors wish to thank Mr D Darcey for technical assistance and Mr P Burrows, Ms M Carter, and Mr D Heliams for anaesthetic assistance.

1 Lovasik JV, Kothe AC, Kergoat H. Comparison of noninvasive methods to derive the mean central retinal artery pressure in man. Optom Vis Sci 1993;70:1005-11.

2 Hedges TR, Weinstein JD, Kassell NF, et al. Correlation of ophthalmodynamometry with ophthalmic artery pressure in the rhesus monkey. Am $\mathcal{F}$ Ophthalmol 1965;60:1098101

3 Williamson TH, Baxter GM, Pyott A, et al. A comparison of Doppler imaging of orbital vessels and other methods of blood flow assessment. Graefes Arch Clin Exp Ophthalmol 1995;233:80-4.

4 Cennamo G, Rosa N, De Palma L, et al. Echographic and ophthalmodynamometric study in the empty sella synophthalmodynamometric study in the
drome. Ophthalmologica 1993;206:29-32

5 Van der Werff TJ. The pressure measured in ophthalmodynamometry. Arch Ophthalmol 1972;87:290-2.

6 Mullie MA, Kirkham TH. Ophthalmodynamometry revisited. Can f Ophthalmol 1983;18:165-8.

7 Duke-Elder WS. The ocular circulation: its normal pressure relationships and their physiological significance. $\mathrm{Br} \mathcal{F} \mathrm{Oph}$ thalmol 1926;10:513-72.

8 Grunwald JE, Furubayashi C. Effect of topical timolol maleate on the ophthalmic artery blood pressure. Invest Ophthalmol Vis Sci 1989;30:1095-100.

9 Barnes RW. Other noninvasive techniques in cerebrovascular disease. In: Bernstein EF, ed. Vascular diagnosis. St Louis: Mosby Year Book, 1993:399-400.

10 Weinberger J. Clinical applications of noninvasive carotid artery testing. F Am Coll Cardiol 1985;5:137-48.

11 Weibers DO, Folger N, Forbes GS, et al. Ophthalmodynamometry and ocular pneumoplethysmography for detecnamometry and ocular pneumoplethysmography for detec-
tion of carotid occlusive disease. Arch Neurol 1982;39:6901.

$12 \mathrm{Hu} \mathrm{HH}$, Sheng WY, Yen MY, et al. Colour doppler imaging of orbital arteries for detection of carotid occlusive disease. Stroke 1993; 24:1196-203.

13 Robinson F, Riva CE, Grunwald JE, et al. Retinal blood flow autoregulation in response to an acute increase in blood pressure. Invest Ophthalmol Vis Sci 1986;27:722-6

14 AbuRahma AF, Robinson PA, Short Y, et al. Cross-filling of circle of Willis and carotid stenosis by angiography, duplex ultrasound, and oculopneumoplethysmography. Am ₹ Surg 1995;169:308-12.

15 Holladay JT, Arnoult JB, Ruiz RS. Comparative evaluation of current ophthalmodynamometers. Am f Ophthalmol 1979; 87:665-74.

16 Zeiher A. Microcirculation. In: Drance SM, ed. Optic nerve in glaucoma. Amsterdam: Kugler Publications, 1995:247-

17 DeLano FA, Schmid-Schonbein GW, Skalak TC, et al. Penetration of the systemic blood pressure into the microvasculature of rat skeletal muscle. Microvasc Res 1991;41: 92-110. 
18 Gore RW. Pressures in cat mesenteric arterioles and capillaries during changes in systemic arterial blood

9 Morgan WH, Yu DY, Cooper RL, et al. The influence of the lamina cribrosa tissue pressure gradient. Invest Ophthalmol Vis Sci 1995;36:116372.

20 Fung YC. Biodynamics: circulation. New York: SpringerVerlag, 1984:132-4.

21 Eikelboom BC. Physiologic principles of ocular pneumoplethysmography. In: Bernstein EF, ed. Vascular diagnosis. St Louis: Mosby Year Book, 1993:176-80.

22 Drzewiecki G, Hood R, Apple H. Theory of the oscillomet- ric maximum and the systolic and diastolic detection ratios. Ann Biomed Eng 1994;22:88-96.

23 Best M, Blumenthal M, Futterman HA, et al. Critical closure of intraocular blood vessels. Arch Ophthalmol 1969; 82:385-92.

24 Morgan WH, Yu DY, Cooper RL, et al. Retinal artery and vein pressures in the dog and their relationship to aortic, intraocular and cerebrospinal fluid pressures. Microvasc Res 1997;53:211-21.

25 Eikelboom BC. Oculoplethysmography and ocular pneumoplethysmography. In: Bernstein EF, ed. Vascular diagnosis. St Louis: Mosby Year Book, 1993:403-7. 